\title{
Adaptation and visual search in mammographic images
}

\author{
Elysse Kompaniez-Dunigan • Craig K. Abbey • \\ John M. Boone • Michael A. Webster
}

Published online: 27 February 2015

(C) The Psychonomic Society, Inc. 2015

\begin{abstract}
Radiologists face the visually challenging task of detecting suspicious features within the complex and noisy backgrounds characteristic of medical images. We used a search task to examine whether the salience of target features in x-ray mammograms could be enhanced by prior adaptation to the spatial structure of the images. The observers were not radiologists, and thus had no diagnostic training with the images. The stimuli were randomly selected sections from normal mammograms previously classified with BIRADS Density scores of "fatty" versus "dense," corresponding to differences in the relative quantities of fat versus fibroglandular tissue. These categories reflect conspicuous differences in visual texture, with dense tissue being more likely to obscure lesion detection. The targets were simulated masses corresponding to bright Gaussian spots, superimposed by adding the luminance to the background. A single target was randomly added to each image, with contrast varied over five levels so that they varied from difficult to easy to detect. Reaction times were measured for detecting the target location, before or after adapting to a gray field or to random sequences of a different set of dense or fatty images. Observers were faster at detecting the targets in either dense or fatty images after adapting to the
\end{abstract}

E. Kompaniez-Dunigan $(\triangle) \cdot$ M. A. Webster

Department of Psychology, University of Nevada, Reno,

Reno, NV 89557, USA

e-mail: ekompaniez@unr.edu

C. K. Abbey

Department of Psychological and Brain Sciences, University of California, Santa Barbara, CA, USA

J. M. Boone

Department of Radiology, University of California, Davis, CA, USA

J. M. Boone

Department of Biomedical Engineering, University of California, Davis, CA, USA specific background type (dense or fatty) that they were searching within. Thus, the adaptation led to a facilitation of search performance that was selective for the background texture. Our results are consistent with the hypothesis that adaptation allows observers to more effectively suppress the specific structure of the background, thereby heightening visual salience and search efficiency.

Keywords Adaptation · Aftereffects · Visual search $\cdot$ Medical image perception $\cdot$ Visual salience

The process of "reading" medical images often requires that radiologists search images for subtle abnormalities. This involves detecting anomalies or suspicious features within images that have complex and noisy background characteristics. Moreover, these characteristics often reflect unnatural image statistics that are therefore themselves anomalous relative to the normal visual diet of an observer. Thus, visual training for medical image diagnosis is obviously fundamentally important for developing the requisite skills for reading and interpreting the images.

In the effort to improve detection accuracy and efficiency, extensive research has investigated visual search in medical images. Radiologists are able to rapidly extract a great deal of information from these images (Drew, Evans, Võ, Jacobson, \& Wolfe, 2013; Krupinski, 1996; Kundel \& Follette, 1972; Kundel \& Nodine, 1975; Kundel, Nodine, Krupinski, \& Mello-Thomas, 2008; Mugglestone, Gale, Cowley, \& Wilson, 1995). The initial inspection gives rise to a global impression that then acts as a filter to direct attention to areas of further interest (Kundel, Nodine, Thickman, \& Toto, 1987; Swensson, 1980). Importantly, this initial stage enables readers to compare the image under inspection to prior knowledge of normal structures within the image. When given a short duration $(200 \mathrm{~ms})$ to inspect chest radiographs, 
radiologists performed surprisingly well, with approximately $70 \%$ correct classifications of images as normal versus abnormal (Kundel \& Nodine, 1975). This brief presentation allowed for only one initial eye fixation, revealing that a great deal of information is extracted from the image in parallel, and that readers are able to make diagnostic decisions on the basis of this initial representation. This was also observed when radiologists were tasked with inspecting mammogram images (Mugglestone et al., 1995), with approximately $67 \%$ of the locations containing cancers being located within $1 \mathrm{~s}$ (Kundel et al., 2008). The ability to make a diagnostic decision on the basis of a rapid global impression is likely due to the expert radiologist's comparing the image under inspection to that of internal representations of what is expected (normal) in the image and what is abnormal. This is supported by changes in search patterns through experience and training (Kundel \& Follette, 1972), and is further evidenced by the fact that experienced readers detect targets more quickly (Krupinski, 1996) and more accurately than less-experienced readers (Donovan \& Litchfield, 2013; Snowden, Davies, \& Roling, 2000). Furthermore, novices improve through training, indicating that the detection of targets can be enhanced through perceptual learning (Snowden et al., 2000). Search performance also varies with target prevalence: If target prevalence is low (Gur et al., 2004), which is the case in routine mammography screenings, detection rates decrease (Wolfe, Horowitz, \& Kenner, 2005; Wolfe et al., 2007; Wolfe \& Van Wert, 2010). This is due to the fact that rare targets are often missed because participants do not expect them in a large proportion of the images.

In this study, we examined the influence of sensory adaptation on visual search: a form of short-term visual "training" that has received very little attention in the medical image perception literature. Adaptation occurs throughout the visual pathway and continuously adjusts sensitivity to the stimuli that observers are currently exposed to (Webster, 2011). The neural response changes induced by adaptation affect not only the appearance of images, but also visual performance- how accurately or quickly observers can make judgments or detect information within the images. Adaptation has been found to improve discrimination tasks across a wide range of stimuli, including, but not limited to, orientation, speed judgments, tilt, and contrast (Abbonizio, Langley, \& Clifford, 2002; Clifford, Wyatt, Arnold, Smith, \& Wenderoth, 2001; Kristjánsson, 2011), though these improvements are not always robust (Clifford et al., 2007). Many of these studies have investigated enhancements in threshold discriminations, but adaptation might also lead to improvements in suprathreshold tasks such as visual search. McDermott and colleagues (McDermott, Malkoc, Mulligan, \& Webster, 2010) investigated whether adaptation could increase the salience of chromatic targets presented within a chromatically varying background. They found that observers were better able to detect novel colored targets following adaptation to a background color distribution, suggesting that adaptation enhances the salience of the target relative to the background. Adaptation has also been found to improve accuracy and decrease reaction times (RTs) when searching for novel orientations (Wissig, Patterson, \& Kohn, 2013). These studies suggest that adaptation may serve to highlight the salience of novel stimulus properties by discounting or reducing the salience of the ambient or expected properties of the images.

In the present study, we investigated whether adaptation could enhance the salience of a target in medical images, allowing observers to more rapidly detect abnormalities in radiological scans. Recently we have shown that adaptation to medical images produces robust and rapid aftereffects in the perceived texture of mammogram images (Kompaniez, Abbey, Boone, \& Webster, 2013). Specifically, adaptation to dense or fatty images induced large and selective changes in the perceived texture of mammograms. Here we investigated whether this adaptation also influences the ability to detect abnormalities (simulated masses) within the mammograms. To assess this, we tested "lay" observers with no training in medical diagnosis, using a standard visual search task in which observers had to locate targets superimposed on backgrounds taken from actual mammogram sections. This task did not simulate many of the factors involved when actual radiologists conduct actual readings, but instead provided a controlled stimulus environment that allowed us to examine, as a proof of concept, whether adaptation to the characteristic properties of mammograms could facilitate a target detection task like those confronting radiologists.

\section{Materials and methods}

\section{Observers}

Ten observers with normal or corrected-to-normal visual acuity participated in the experiments. The observers included authors E.K. and M.W., as well as eight students who were naive to the purpose of the study. None of the observers had medical training, and thus none had prior experience in radiological diagnosis. This was done in part in ensure that the task, and thus the potential adaptation, would be novel to the observer. Participation was with written informed consent and followed protocols approved by the university's Institutional Review Board.

Apparatus and stimuli

All stimuli were presented on a calibrated and gammacorrected Sony 500 PS monitor controlled by a Cambridge Research Systems VSG graphics card. The images were taken from actual mammogram images that had been classified 
previously as "dense" or "fatty" by radiologists as part of a clinical report. BIRADS density scores are a standard component of screening mammography and are assigned on a 4point scale (1-Fatty, 2-Scattered Density, 3-Heterogeneously Dense, and 4-Dense) by clinicians. We used images with scores at the extremes of the scale (1-Fatty and 4-Dense) to maximize our ability to detect an adaptation effect. The scans used in our studies came from a population of women who were being recalled to biopsy on the basis of a suspicious finding; however, our images always came from the unaffected breast. All images were deidentified and acquired under an IRB-approved protocol with patient consent on a clinical digital mammography scanner (Hologic Selenia, Bedford MA) at standard dose protocols and with standard display processing.

For the visual search experiments, the stimuli consisted of randomly selected sections corresponding to $800 \times 600$ pixels $(80 \times 60 \mathrm{~mm}$ sections $)$ from the original $2,560 \times 3,328$ pixel images, and were constrained to be fully within the breast region of the image. Sets of these images taken from mammograms classified as dense or fatty served as the adapting stimuli. For the test stimuli, we used similar random sections taken from a different subset of images. These consisted of $20 \mathrm{im}-$ ages from mammograms classified as dense and 20 images classified as fatty. To control for variations in average luminance and contrast across the images, all of the images were adjusted to have a constant mean $\left(37 \mathrm{~cd} / \mathrm{m}^{2}\right)$ and RMS contrast $(0.38)$. For the test images, the targets were simulated "lesions" corresponding to incremental Gaussian spots (SD $=0.18 \mathrm{deg}$ ), superimposed by adding the spot's luminance to the background (Fig. 1). Gaussian spots are commonly used to simulate targets in noise or medical imaging studies (Burgess, Li, \& Abbey, 1997; Burgess, Wagner, Jennings, \& Barlow, 1981; Myers \& Barrett, 1987; Park, Gallas, Badano, Petrick, \& Myers, 2007; Rolland \& Barrett, 1992; Wagner \& Brown, 1985), to provide a simple and well-circumscribed stimulus that does not require training and that can be easily manipulated to vary the difficulty of the search task. The location of the target was chosen randomly, with the constraint that it did not fall within $0.55 \mathrm{deg}$ to the left or right of the

\section{Mammograms with added targets (circled)}

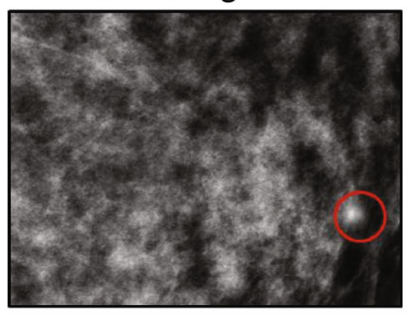

Dense Tissue

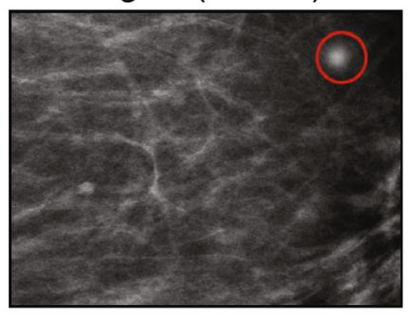

Fatty Tissue
Fig. 1 Examples of test stimuli for the visual search task. Simulated masses (circled to show their location in the figure) were added at random locations to image sections from actual mammogram images that had been classified as fatty or dense as part of the clinical report center of the background, and thus could be readily localized to the left or right side of the image. Finally, the target contrast was varied in separate images over five levels (corresponding to linearized D/A increments of $70,90,110,130,150)$ so that detection varied from easy to difficult (Fig. 2).

\section{Procedure}

Observers viewed the display binocularly in a darkened room from a distance of $260 \mathrm{~cm}$, at which the screen subtended 6.6 $\times 8.75 \mathrm{deg}$. In the pre-adapt conditions, observers initially adapted to a uniform gray field for $30 \mathrm{~s}$, followed by the visual search task. In the adapt conditions, the search task was instead preceded by $5 \mathrm{~min}$ of adaptation to fatty or dense images, during which the observer free-viewed the adapting sequence without maintaining fixation. In both the pre-adapt and adapt conditions, the start of the search sequence was signaled by a tone $5 \mathrm{~s}$ prior to the presentation of the test stimuli. The adapting stimuli filled the monitor screen and cycled randomly through ten samples of either dense or fatty tissue at a rate of $250 \mathrm{~ms}$ per image, to ensure that participants were adapting to the characteristic texture of the mammograms rather than to a single image.

During the search task, a test image was randomly selected from either the dense or the fatty set, and the onset of the image was accompanied by a tone. Observers used a button press to respond as quickly as possible whether the target fell on the left or the right side of the monitor screen. A separate button was also available to respond if they could not find the target (in which case the trial was treated as an incorrect response). The test image remained on the screen until a response was made. This was then followed by 4-s re-adapt period to the gray screen or a new random sequence of the adapting images, followed by the presentation of the next test image.
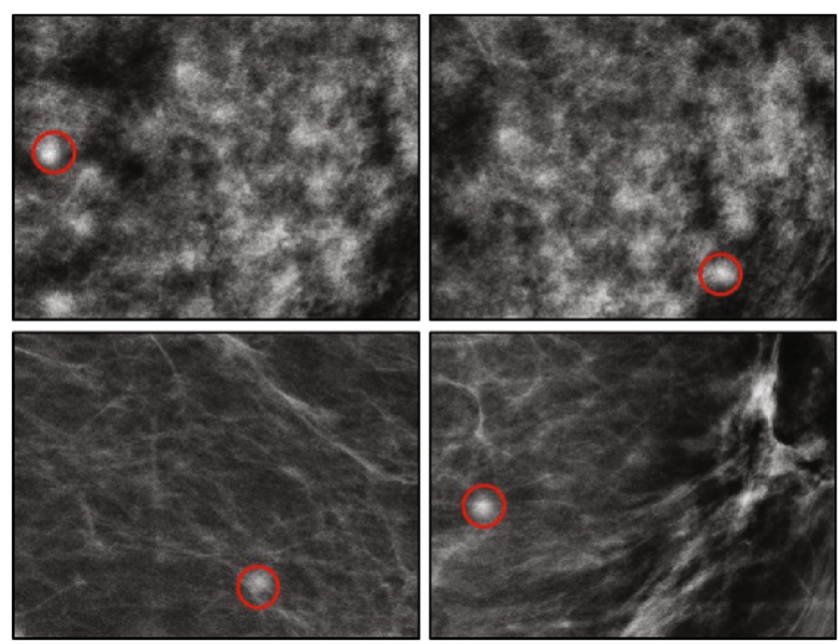

Fig. 2 Examples of different test stimuli with varying target contrasts (circled) 
Observers were tested in four separate daily sessions. In each, they first conducted two pre-adapt runs (twice searching for the targets in all 40 test images while adapted to a gray background), followed by four repeated runs for either the dense or the fatty adapt condition (with adaptation to only the dense or the fatty set on a given day). The order of the test images was randomized during each run, and the two adapt conditions (dense and fatty) were counterbalanced across days. This sequence resulted in eight repeated settings for each of the 40 test images in each of the three adapting conditions. To avoid learning of the repeated test images or target locations, the test stimuli were shown either in their original orientation, mirrored along the horizontal or vertical axes, or rotated $180 \mathrm{deg}$ (with two repetitions of each of these four variants). For each observer's settings, we calculated the median RT for each image on the basis of all trials in which the target location was identified correctly. All reported results are based on the settings averaged across the observers.

\section{Results}

Search times under neutral (gray field) adaptation

RTs varied widely across the different test images ( $\mathrm{min}=514$ $\mathrm{ms}, \max =1948 \mathrm{~ms}$ for correct responses). This was as expected, since the target contrast was intentionally varied over a wide range to ensure a range of difficulty in the task. To quantify the effect of contrast, for each test we calculated a local measure of contrast by comparing the relative luminance of the target to a Gaussian-weighted average of the background luminance (centered at the target location, but with twice the spatial standard deviation of the target). Figure 3 shows that this measure of local contrast can partly capture the differences in search times across the different images $(r=$ $-.57, p<.01)$.

Search times under adaptation to dense images

The remaining figures illustrate the effects of adaptation on the search times. Figure 4 compares the search times before and after adapting to the dynamic array of dense images. Again, each point plots the average RT for detecting one of the 40 targets, based on averaging the median RTs from each observer's repeated settings (eight per image) for a given adapt condition. Adaptation to the dense images consistently improved detection when searching for targets in dense images (decreasing RTs by an average of $198 \mathrm{~ms}$, or $13.8 \%$ ), but did so less for the fatty images (mean change $=115 \mathrm{~ms}$, or $7.5 \%$ ). This suggests that adaptation was selective for the image type. This was verified by a sign test comparing the pre-

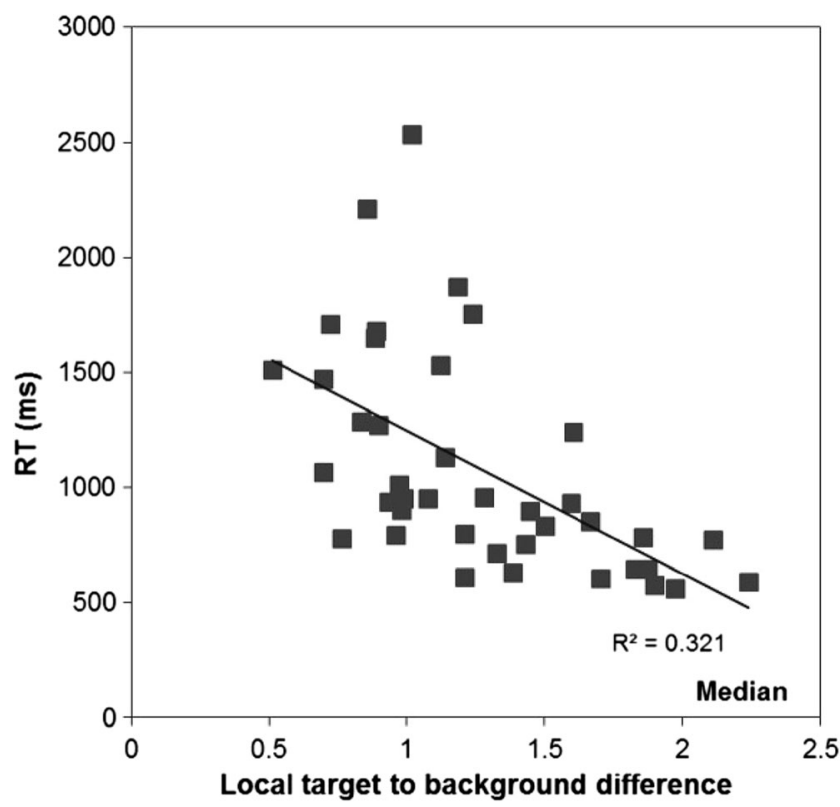

Fig. 3 Average "pre-adapt" reaction times (RTs) as a function of the contrast of the target relative to the local background. Each point plots the mean RT for one of the 40 test images, based on averaging the median RTs for the ten observers under neutral adaptation to the gray background

and post-adapt $\mathrm{RTs}(Z=3.8, p<.0001$, for dense; $Z=0.22, p=$ .413 , for fatty). Similar effects were also found when analyzing the results only for the eight naïve observers $(Z=2.91, p=$ .0013 , for dense; $Z=0.92, p=.1796$, for fatty). Notably, the overall accuracy remained between $70 \%$ and $80 \%$ correct for

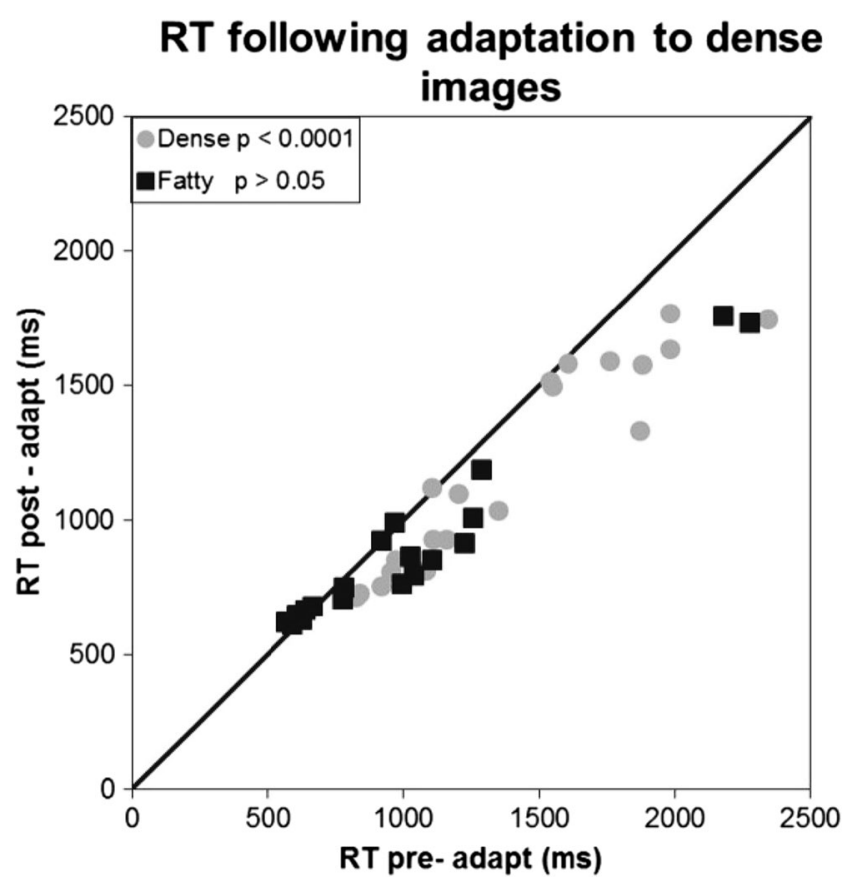

Fig. 4 Average reaction times (RTs) for detecting targets within dense or fatty images, before (pre-adapt) or after (post-adapt) adaptation to dense images. Adaptation to dense images significantly improved detection when searching within dense, but not fatty, images 
all conditions, and in particular did not differ significantly across the adapting conditions. Thus, the improvements in search times for the dense images did not reflect a speedaccuracy trade-off, suggesting that they instead reflect actual changes in search efficiency.

Search times under adaptation to fatty images

Figure 5 plots the comparable results for the condition in which observers instead adapted to the fatty image set. This led to similar results, in that observers' search times were reduced following adaptation to fatty images (mean change $=180 \mathrm{~ms}$, or a $13 \%$ improvement), but not to dense ones (mean change $=59 \mathrm{~ms}$, or $4.6 \%$ ). These effects were again assessed by a sign test (for all observers: $Z=2.91, p=.0013$, for fatty; $Z=1.12, p=.132$, for dense; for the eight naïve observers: $Z=3.35, p=.0002$, for fatty; $Z=0.67, p=.252$, for dense). Again this reduction in RTs was not due to a speedaccuracy trade-off, since accuracy remained similar.

In both cases, the RTs improved only when the test backgrounds and adapt backgrounds were drawn from the same class of images. Thus, the effects of the adaptation were not general, but instead were selective for the specific characteristics that distinguished the dense and fatty images. This result was based on comparisons of each aftereffect relative to the pre-adapt baseline. However, as a further test of this selectivity, we also directly compared the search times for the dense or fatty test images when observers were adapted to either the

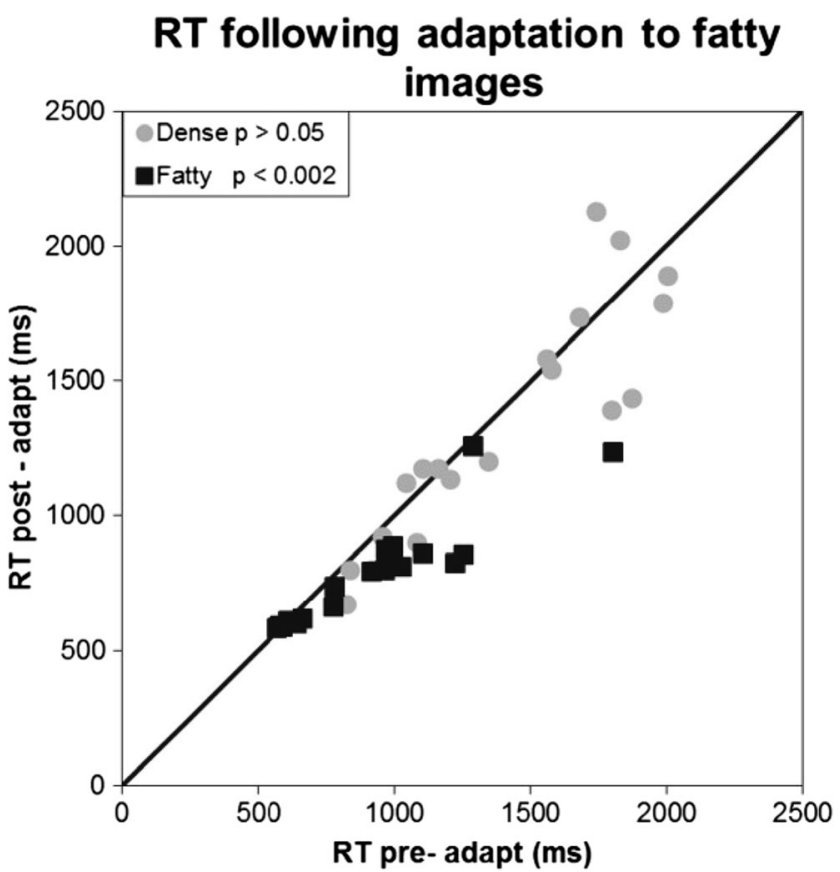

Fig. 5 Average reaction times (RTs) for detecting targets within dense or fatty images, before (pre-adapt) or after (post-adapt) adaptation to fatty images. Adaptation to fatty images significantly improved detection when searching within fatty, but not dense, images dense or the fatty images Again, this analysis confirmed that adaptation selectively facilitated detection of the targets when searching within the same image type as the adapt condition $(Z=3.35, p=.0002$, for dense; $Z=2.46, p=.006$, for fatty).

\section{Discussion}

Our results demonstrate that prior exposure to dense or fatty images facilitates search for target "masses" embedded in mammogram images. Moreover, this enhancement is specific to the adapting image type (dense or fatty), and thus reflects selective performance improvements rather than simple generic learning. This selectivity is consistent with the selectivity that we observed previously in the appearance of the mammograms after adaptation (Kompaniez et al., 2013). There we showed that adapting to the dense (or fatty) images causes an intermediate image to appear more fatty (or dense). Thus, the adaptation itself appears to be selective for the textural properties that differentiate the two classes of mammograms.

But why should this selective adaptation impact visual search? As we noted in the introduction, one putative role for sensory adaptation is to discount the expected properties of the world in order to enhance or draw attention to more novel properties (Webster, 2014). In fact, we previously had also observed aftereffects consistent with this account in the appearance of the images. Specifically, adaptation to the dense or fatty images caused the adapting images themselves to appear less fatty or less dense over time (Kompaniez et al., 2013). This suggests that the textural characteristics of the backgrounds became less distinct or more neutral in appearance with prolonged viewing, consistent with a renormalization of perception with adaptation. Similar normalization effects have been observed across multiple stimulus domains, including color (Webster, 2011), blur (Elliott et al., 2011), and faces (Rhodes et al., 2005; Webster \& MacLeod, 2011).

If this "desensitization" affects the background more than the target, a consequence of the adaptation is that it will increase the effective signal-to-noise ratio of the target, and thus enhance its salience. Again, effects of this kind have been observed previously when adapting to fairly simple stimulus dimensions, such as a distribution of colors (McDermott et al., 2010) or arrays of oriented elements (Wissig et al., 2013). They have also been predicted from analyses in which images are processed to simulate the perceptual consequences of very long-term adaptation to specific environments (Webster, 2014). Here we have shown that these effects can potentially also arise over very short timescales within the complex and challenging task of an individual inspecting a mammogram.

One way that adaptation could plausibly enhance target salience is by functioning to "discount" the structure of the 
adapting background (Webster, 2011). A decreased sensitivity to extant properties of the world could then serve to draw attention to stimuli with different properties, thus highlighting novel features within the visual scene. In images with welldefined properties, such as distributions of colors or orientations, it is straightforward to define in what ways a target is novel from the background. However, with more complex and naturalistic patterns it is less obvious, and in particular, we cannot quantify how a Gaussian target differs from a dense or fatty background. Nevertheless, our results are consistent with adaptation acting selectively on the textural characteristics of the backgrounds, relative to the targets.

The present results are clearly limited to highly controlled and unnatural viewing contexts that do not capture many of the factors that are likely to impact how and to what extent adaptation might be manifest during the actual practice of medical image inspection by expert radiologists. The search improvements with adaptation nevertheless reinforce the idea that adaptation can, in fact, selectively adjust for the characteristic structure of dense or fatty mammograms (Kompaniez et al. 2013), and they further show that these adjustments can affect visual performance. Moreover, it is increasingly evident that the processes of adaptation are themselves routinely engaged in natural viewing, and thus can affect most if not all perceptual judgments (Webster, 2011). Thus, these results have potential relevance and practical implications for realworld medical image inspection, though whether the impact in actual clinical practice is significant remains to be tested. Radiologists use very diverse techniques when searching within and classifying mammograms images. For example, readers may scan images in idiosyncratic ways (Krupinski, 1996), and there is relatively little standardization of inspection protocols. Moreover, we know of no attempts to order mammographic images in a batch reading environment (Burnside, Park, Fine, \& Sisney, 2005; Ghate et al., 2005) on the basis of the image properties, to reduce fatigue or reading time or to increase accuracy. If adaptation effects like those shown here persist across image reading times, there may be a benefit to ordering images on the basis of a predetermined estimate of the mammographic density. Specifically, our study suggests that, depending on which images they have viewed in the immediate past, radiologists may be in either a better or a less optimized state of adaptation to detect tumors or lesions within the tissue shown in the current image. This suggests that ordering images by their density type could potentially increase search efficiency, which might also reduce fatigue. Finally, to the extent that the nature of these adaptation effects and the relevant visual structure of radiological images could be appropriately modeled, it should in principle be possible to develop image-processing models to simulate how medical images should appear to observers after they are adapted (in the same way that these models have been developed to simulate the consequences of theoretically optimal color adaptation; Webster, 2014). That is, mammogram images could be "pre-adapted" so that they are optimized for the visual system of the radiologist, removing the time and effort required for the radiologist to instead adapt to the image.

Author Note This research was supported by National Institutes of Health Grants R01 EY-10834, awarded to M.A.W. and R01 EB002138, awarded to J.M.B.

\section{References}

Abbonizio, G., Langley, K., \& Clifford, C. W. G. (2002). Contrast adaptation may enhance contrast discrimination. Spatial Vision, 16, 4558.

Burgess, A. E., Li, X., \& Abbey, C. K. (1997). Visual signal detectability with two noise components: Anomalous masking effects. Journal of the Optical Society of America A, 14, 2420-2442.

Burgess, A. E., Wagner, R. F., Jennings, R. J., \& Barlow, H. B. (1981). Efficiency of human visual signal discrimination. Science, 214, 9394.

Burnside, E. S., Park, J. M., Fine, J. P., \& Sisney, G. A. (2005). The use of batch reading to improve the performance of screening mammography. American Journal of Roentgenology, 185, 790-796.

Clifford, C. W., Webster, M. A., Stanley, G. B., Stocker, A. A., Kohn, A., Sharpee, T. O., \& Schwartz, O. (2007). Visual adaptation: neural, psychological and computational aspects. Vision Research, 47, 3125-3131.

Clifford, C. W., Wyatt, A. M., Arnold, D. H., Smith, S. T., \& Wenderoth, P. (2001). Orthogonal adaptation improves orientation discrimination. Vision Research, 41, 151-159.

Donovan, T., \& Litchfield, D. (2013). Looking for cancer: Expertise related differences in searching and decision making. Applied Cognitive Psychology, 27, 43-49. doi:10.1002/acp.2869

Drew, T., Evans, K., Võ, M. L.-H., Jacobson, F. L., \& Wolfe, J. M. (2013). Informatics in radiology: What can you see in a single glance and how might this guide visual search in medical images? RadioGraphics, 33, 263-274. doi:10.1148/rg.331125023

Elliott, S. L., Georgeson, M. A., \& Webster, M. A. (2011). Response normalization and blur adaptation: Data and multi-scale model. Journal of Vision, 11(2), 7. doi:10.1167/11.2.7

Ghate, S. V., Soo, M. S., Baker, J. A., Walsh, R., Gimenez, E. I., \& Rosen, E. L. (2005). Comparison of recall and cancer detection rates for immediate versus batch interpretation of screening mammograms 1 . Radiology, 235, 31-35.

Gur, D., Sumkin, J. H., Rockette, H. E., Ganott, M., Hakim, C., Hardesty, L., \& Wallace, L. (2004). Changes in breast cancer detection and mammography recall rates after the introduction of a computeraided detection system. Journal of the National Cancer Institute, 96, 185-190. doi:10.1093/jnci/djh067

Kompaniez, E., Abbey, C. K., Boone, J. M., \& Webster, M. A. (2013). Adaptation aftereffects in the perception of radiological images. PLoS ONE, 8, e76175. doi:10.1371/journal.pone.0076175

Kristjánsson, Á. (2011). The functional benefits of tilt adaptation. Seeing and Perceiving, 24, 37-51. doi:10.1163/187847511X555283

Krupinski, E. A. (1996). Visual scanning patterns of radiologists searching mammograms. Academic Radiology, 3, 137-144.

Kundel, H. L., \& Follette, P. S. (1972). Visual search patterns and experience with radiological images. Radiology, 103, 523-528.

Kundel, H. L., \& Nodine, C. F. (1975). Interpreting chest radiographs without visual search. Radiology, 116, 527-532.

Kundel, H. L., Nodine, C. F., Krupinski, E. A., \& Mello-Thomas, C. (2008). Using gaze-tracking data and mixture distribution analysis 
to support a holistic model for the detection of cancers on mammograms. Academic Radiology, 15, 881-886.

Kundel, H. L., Nodine, C. F., Thickman, D., \& Toto, L. (1987). Searching for lung nodules a comparison of human performance with random and systematic scanning. Investigative Radiology, 22, 417-422.

McDermott, K. C., Malkoc, G., Mulligan, J. B., \& Webster, M. A. (2010). Adaptation and visual salience. Journal of Vision, 10(13), 17. doi: $10.1167 / 10.13 .17$

Mugglestone, M. D., Gale, A. G., Cowley, H. C., \& Wilson, A. R. M. (1995). Diagnostic performance on briefly presented mammographic images. Medical Imaging 1995: Image Perception (Proceedings of SPIE 2436, p. 106). SPIE. doi:10.1117/12.206840

Myers, K. J., \& Barrett, H. H. (1987). Addition of a channel mechanism to the ideal-observer model. Journal of the Optical Society of America A, 4, 2447-2457.

Park, S., Gallas, B. D., Badano, A., Petrick, N. A., \& Myers, K. J. (2007). Efficiency of the human observer for detecting a Gaussian signal at a known location in non-Gaussian distributed lumpy backgrounds. Journal of the Optical Society of America A, 24, 911-921.

Rhodes, G., Robbins, R., Jaquet, E., McKone, E., Jeffery, L., \& Clifford, C. W. (2005). Adaptation and face perception-How aftereffects implicate norm based coding of faces. In C. W. Clifford \& G. Rhodes (Eds.), Fitting the mind to the world: Adaptation and aftereffects in high-level vision (pp. 213-240). Oxford, UK: Oxford University Press.

Rolland, J. P., \& Barrett, H. H. (1992). Effect of random background inhomogeneity on observer detection performance. Journal of the Optical Society of America A, 9, 649-658.

Snowden, P. T., Davies, I. R., \& Roling, P. (2000). Perceptual learning of the detection of features in X-ray images: A functional role for improvements in adults' visual sensitivity? Journal of Experimental Psychology: Human Perception and Performance, 26, 379-390.

Swensson, R. G. (1980). A two-stage detection model applied to skilled visual search by radiologists. Perception \& Psychophysics, 27, 1116.

Wagner, R. F., \& Brown, D. G. (1985). Unified SNR analysis of medical imaging systems. Physics in Medicine and Biology, 30, 489.

Webster, M. A. (2011). Adaptation and visual coding. Journal of Vision, 11(5), 3:1-23. doi:10.1167/11.5.3

Webster, M. A. (2014). Probing the functions of contextual modulation by adapting images rather than observers. Vision Research, 104, 68 79.

Webster, M. A., \& MacLeod, D. I. (2011). Visual adaptation and face perception. Philosophical Transactions of the Royal Society B, 366, $1702-1725$.

Wissig, S. C., Patterson, C. A., \& Kohn, A. (2013). Adaptation improves performance on a visual search task. Journal of Vision, 13(2), 6. doi: 10.1167/13.2.6

Wolfe, J. M., Horowitz, T. S., Van Wert, M. J., Kenner, N. M., Place, S. S., $\&$ Kibbi, N. (2007). Low target prevalence is a stubborn source of errors in visual search tasks. Journal of Experimental Psychology: General, 136, 623-638. doi:10.1037/0096-3445.136.4.623

Wolfe, J. M., Horowitz, T. S., \& Kenner, N. M. (2005). Rare items often missed in visual searches. Nature, 435, 439-440. doi:10.1038/ 435439 a

Wolfe, J. M., \& Van Wert, M. J. (2010). Varying target prevalence reveals two dissociable decision criteria in visual search. Current Biology, 20, 121-124. doi:10.1016/j.cub.2009.11.066 